\title{
Amorphous-amorphous transition in glassy polymers subjected to cold rolling studied by means of positron annihilation lifetime spectroscopy
}

\author{
D. Cangialosi ${ }^{\text {a) }}$ \\ Department of Polymer Materials and Engineering, Delft University of Technology, Julianalaan 136, \\ 2628 BL Delft, The Netherlands and Dutch Polymer Institute, P.O. Box 902, 5600 AX Eindhoven, \\ The Netherlands \\ M. Wübbenhorst \\ Department of Polymer Materials and Engineering, Delft University of Technology, Julianalaan 136, \\ 2628 BL Delft, The Netherlands \\ H. Schut and A. van Veen ${ }^{\text {b) }}$ \\ Interfaculty Reactor Institute, Delft University of Technology, Mekelweg 15, 2629 JB Delft, The Netherlands \\ S. J. Picken \\ Department of Polymer Materials and Engineering, Delft University of Technology, Julianalaan 136, \\ 2628 BL Delft, The Netherlands and Dutch Polymer Institute, P.O. Box 902, 5600 AX Eindhoven, \\ The Netherlands
}

(Received 7 June 2004; accepted 9 November 2004; published online 26 January 2005)

In this study, polycarbonate (PC) and polystyrene (PS) are subjected to plastic deformation by means of cold rolling and the resulting variation of the free volume and its subsequent time evolution after rolling is investigated by means of positron annihilation lifetime spectroscopy (PALS). The value of the long lifetime component that is attributed to the decay of ortho-positronium $\left(\tau_{\mathrm{o}-\mathrm{Ps}}\right)$ and its intensity $\left(I_{\mathrm{o}-\mathrm{Ps}}\right)$ are used to characterize, respectively, the size and the concentration of the free-volume holes. In addition to the PALS experiments, the effect of plastic deformation on the dynamic tensile modulus is investigated. The PALS results show that both for well-aged PC and PS an increase of $\tau_{\mathrm{o}-\mathrm{Ps}}$ and a decrease of $I_{\mathrm{o}-\mathrm{Ps}}$ occur upon plastic deformation. During the subsequent aging, $\tau_{\mathrm{o}-\mathrm{Ps}}$ tends to return to the value assumed before plastic deformation, while $I_{\mathrm{o}-\mathrm{Ps}}$ remains constant with time. These results corroborate the idea of an amorphous-amorphous transition, rather than that of a "mechanical rejuvenation" as proposed in the past to explain the ability of plastic deformation to reinitiate physical aging. Finally, a linear relation between the size of the free-volume holes and the dynamic tensile modulus is found, which suggests that the stiffness of amorphous glassy polymers is fully determined by their nanoscopic structure. (C) 2005 American Institute of Physics. [DOI: 10.1063/1.1844396]

\section{INTRODUCTION}

The physics of the glassy state has been the subject of a number of studies throughout the past decades. In particular, great interest has been devoted to the slow evolution of the thermodynamic properties that occurs after cooling from the equilibrium melt. Increasing relaxation times result from a temperature reduction and the glass former cannot equilibrate during the time scale of feasible experiments. The structural changes introduced by this phenomenon, known as "physical aging," can be erased by increasing the temperature above the glass transition of the glass former. This effect is generally known as "thermal rejuvenation."1 However, it was also proposed that application of large stresses in the glassy state can have the same effect of erasure of the thermomechanical history of the sample. ${ }^{1,2}$ In this case, a "mechanically rejuvenated" glass is obtained with properties resembling those of a thermally rejuvenated glass.

\footnotetext{
${ }^{a}$ Present address: Fundacion Donostia International Physics Center, Paseo Manuel de Lardizabal, 4-20018 San Sebastian, Spain.

${ }^{b)}$ Deceased.
}

Recently, McKenna, ${ }^{3}$ discussing a number of experiments performed on materials stressed above the yield point, showed that polymeric glasses seem to end up into a new state, which is different from the one obtained after thermal rejuvenation above $T_{g}$. In particular, he showed that the application of stresses above the yield point generates a calorimetric response in the neighborhood of $T_{g}$, which is different from that of thermally rejuvenated samples. Similar to the case of thermal rejuvenation, the exothermic peak appearing in the enthalpy-temperature plot after aging disappears after mechanical rejuvenation. However, in the latter case a subglass transition minimum in the enthalpy appears. In addition, the decrease over time of the yield stress after thermal and mechanical rejuvenation differs greatly, particularly in the equilibrium values of the yield stress. ${ }^{4}$ Based on these observations, it was proposed that application of large stresses may lead to a sort of amorphous-amorphous transition. ${ }^{3}$ Theoretically, this idea is supported by the concept of the "potential energy landscape." Using this concept, Malandro and Lacks ${ }^{6}$ and Gagnon et al. ${ }^{7}$ describe yielding as the promotion of mechanical instabilities by means of mo- 
lecular simulation on particles interacting through the Weber and Stillinger potential. ${ }^{8}$ This, in turn, drives the system to a different potential energy minimum. Monte Carlo simulation applied to plastically deformed silica, shows such a jump to a different potential energy minimum, which in this case is explained by a continuous increase of the coordination number from four to six. ${ }^{9,10}$ More evidence for a pressure induced amorphous-amorphous transition in a number of nonpolymeric glasses is reported in a recent review. ${ }^{11}$ By means of atomistic modeling, Jang and $\mathrm{Jo}^{12}$ corroborate the idea of an amorphous-amorphous transition in the case of polymers. They showed that the ratio of trans/gauche/unstable state of the dihedral angles in poly(trimethylene terephthalate) undergoes a substantial change upon stress application.

Interesting results have also been obtained from density measurements. In particular, a number of studies have been reported in the past on cold rolled polycarbonate (PC) and acrylonitrile-butadiene-styrene copolymer, ${ }^{13}$ and more recently on polystyrene (PS). ${ }^{14}$ These studies show an increase or very little changes of the density of the polymers after rolling instead of the expected decrease for a rejuvenated sample.

Though the macroscopic effects induced by the application of large stresses are quite well understood in terms of mechanical and thermodynamic properties, a clear interpretation of these results from a microscopic point of view is still lacking. The purpose of this study is to clarify this aspect by means of positron annihilation lifetime spectroscopy (PALS), which allows the characterization of the freevolume structure of polymers. In this technique, positrons emitted by a radioactive source $\left({ }^{22} \mathrm{Na}\right)$ are injected into the polymer where they annihilate. Apart from direct annihilation, positrons can also pick up an electron before annihilating and form ortho-positronium (o-Ps) or para-positronium (p-Ps). The latter has a very short lifetime (125 ps in vacuum) and is not useful to characterize the free volume of polymers. On the other hand, o-Ps has a lifetime in vacuum of $140 \mathrm{~ns}$ that is strongly reduced in polymers to few nanoseconds depending on the free-volume properties of the sample. ${ }^{15}$ In particular, the measured o-Ps lifetime is related to the size of the free-volume cavity using the following semiempirical equation: ${ }^{16}$

$$
\tau_{\mathrm{o}-\mathrm{Ps}}=\frac{1}{2}\left[1-\frac{R}{R+\Delta R}+\frac{1}{2 \pi} \sin \left(\frac{2 \pi R}{R+\Delta R}\right)\right]^{-1},
$$

where $R$ is the radius of the cavity. Equation (1) describes the o-Ps lifetime and, although derived for an infinitely deep potential well (representing the assumed spherical cavity), it contains the empirical parameter $\Delta R=1.656 \AA$ that allows the overlap between the positron and the electron wave function, which would otherwise not be possible due to the infinity of the potential well. ${ }^{16}$

The intensity of o-Ps $\left(I_{\mathrm{o}-\mathrm{Ps}}\right)$, which is proportional to the probability of positronium formation, has been related in the past to the density of free-volume holes in the polymer. ${ }^{17}$ However, it was found that $I_{\mathrm{o}-\mathrm{Ps}}$ is also affected by the chemical composition of the polymer, ${ }^{18}$ the source strength and electrostatic charging due to prolonged irradiation, ${ }^{19-21}$ electric fields ${ }^{22,23}$ and visible light. ${ }^{24}$ Although the interpre- tation of $I_{\mathrm{O}-\mathrm{Ps}}$ as a measure for free volume has been questioned, ${ }^{25}$ we believe that, provided that all the previously mentioned effects are carefully eliminated, or at least minimized, a direct relation between $I_{\mathrm{o}-\mathrm{Ps}}$ and the hole density exists. In the present study, we compare the free-volume parameters of systems with (i) exactly the same chemical composition; (ii) no charging or visible light effects; (iii) where the same source has been used for all the experiments; and (iv) where only one sample is used for each experiment to avoid accumulation of charges. Under similar experimental conditions, it was shown in the past that the reduction of $I_{\mathrm{o}-\mathrm{Ps}}$ during physical aging of PC, gives useful information on the evolution of the polymer structure. ${ }^{26,27}$ For this reason, we feel that it is justified to use the following equation to evaluate the variation the free-volume percentage compared to "fresh" samples (in this case before plastic deformation):

$$
\begin{aligned}
\left(\frac{\nu_{f}}{V_{\text {tot }}}-\frac{\nu_{f 0}}{V_{\text {tot. } 0}}\right) \% & \cong \frac{\nu_{f}-\nu_{f 0}}{V_{\text {tot. } 0}} \% \\
& =\Delta f_{\nu} \%=A \frac{4}{3} \pi\left(R^{3} I_{\mathrm{o}-\mathrm{Ps}}-R_{0}^{3} I_{\mathrm{o}-\mathrm{Ps}(0)}\right),
\end{aligned}
$$

where the subscript (0) indicates the free-volume properties of the fresh samples, $V_{\text {tot }}$ is the overall volume, and $A$ is an empirical parameter taken from literature data. The value of $R$ is derived from the lifetime using Eq. (1). Although for large variations of hole density Eq. (2) is not rigorously valid, ${ }^{17}$ we assume a linear relation between the concentration of holes and $I_{\mathrm{o}-\mathrm{Ps}}$ since the variation of $I_{\mathrm{o}-\mathrm{Ps}}$ is small, as it will be seen in the results section. Note that in Eq. (2) the variation of the overall volume was assumed to be negligible compared to the variation of the free volume. For a freevolume percentage of about $7 \%$ and variation of less than $1 \%$, this results in a negligible error.

The free-volume properties as determined by PALS of plastically deformed polymers were studied in the past by Hasan et al. ${ }^{28}$ However, the PALS parameters were determined during plastic deformation and no hint of a new structure obtained in this manner was reported. To the best of our knowledge, no studies exist on the microscopic structure assumed by glassy amorphous polymers after plastic deformation. The purpose of this paper is to determine the microscopic free-volume structure of "mechanically rejuvenated" PC and PS by means of cold rolling and to interpret the results in terms of polymorphism of the glassy state of polymers. The PALS results are supported by dynamicmechanical tests in order to further investigate possible links between free volume and macroscopic mechanical properties.

\section{EXPERIMENT}

\section{A. Materials and sample preparation}

The materials used in this study are a commercial grade PC (Lexan 161) from General Electric Co. and a commercial grade PS (Styron 648) from Dow Chemical with $M_{w}$ $=318490$ and polydispersity of 3.09 .

Cold rolling was carried out on $3.2 \mathrm{~mm}$ thick PS and PC using a two-roller mill. "Well-aged" injection moulded 
samples were employed for the rolling. The thickness reductions investigated in this study were 19\%, 28\%, 33\%, and $41 \%$ for PC, and $20 \%, 29 \%, 35 \%$, and $40 \%$ for PS. It is worth remarking that, for all thickness reductions, all of the samples pass the yield point and reach the plastic deformation region. Samples of about $1 \mathrm{~cm}^{2}$ were used for PALS measurements, whereas tensile bars according to ASTM D 638 were used for dynamic tensile modulus measurements.

\section{B. PALS measurements}

PALS measurements were performed at room temperature using radioactive ${ }^{22} \mathrm{NaCl}$ source with an activity of $10 \mu \mathrm{Ci}$. The duration of each experiment was about $8 \mathrm{~h}$, which is low enough to minimize charging effects. This was recently demonstrated for $\mathrm{PC},{ }^{21}$ while for PS we have performed preliminary experiments that also show negligible charging effects in the time scale of several hours.

Details on the experimental setup are given in a previous paper. ${ }^{26}$ The positron lifetime spectrum $y(t)$ is analyzed using the program POSITRONFIT, which describes the spectrum as a convolution of the instrument resolution function and a finite number of decaying exponentials plus the background as described by the following equation: ${ }^{29}$

$$
y(t)=R(t) \otimes\left(N \sum_{i=1}^{n} I_{i} \lambda_{i} \exp \left(-\lambda_{i} t\right)+B\right) .
$$

Here $R(t)$ is the resolution function of the system, $\lambda_{i}$ is the annihilation rate (inverse of the lifetime component $\tau_{i}$ ), and $B$ is the background signal. All spectra were resolved in three lifetime components: a short one systematically larger than the lifetime in vacuum of p-Ps (125 ps), in agreement with recent studies on delayed positronium formation, ${ }^{30}$ an intermediate one of around 400 ps related to free positron annihilation, and a long component $\left(\tau_{\mathrm{o}-\mathrm{Ps}}\right)$ related to o-Ps annihilation. The ratio of the intensities of p-Ps and o-Ps was systematically higher than the commonly accepted value $(1 / 3)$. This was also shown to be the case in Ref. 30.

\section{Mechanical characterization}

Tensile samples, according to ASTM D638, were cold rolled to a thickness reduction of $10 \%$ for PC and $30 \%$ for PS. Immediately after rolling the specimen was mounted in a MTS 831 servo-hydraulic tensile tester. The dynamic modulus was subsequently measured using a sinusoidal excitation at a frequency of $1 \mathrm{~Hz}$ with an amplitude of $0.2 \%$ strain. To avoid buckling the sample was prestrained by $0.3 \%$. The modulus determined was averaged over 20 cycles. After this measurement the sample was unloaded and left undisturbed for a certain period of time. Subsequently the modulus measurement was repeated followed again by unloading and a period of undisturbed aging. In this way the evolution of the dynamic tensile modulus was monitored stepwise over a period of $\approx 15 \mathrm{~h}$.

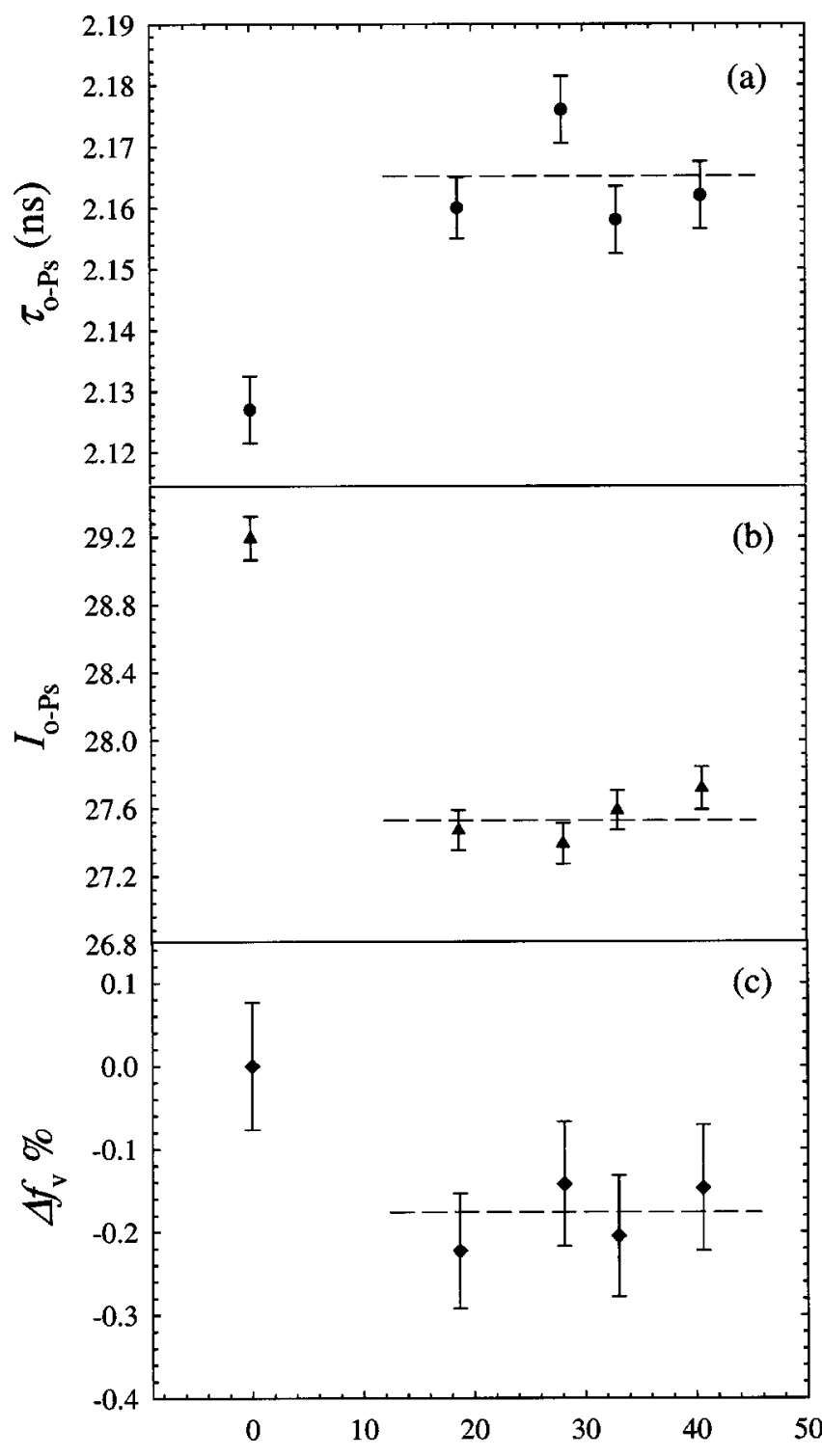

FIG. 1. Effect of the percentage of thickness reduction on the PALS parameters of PC: (a) $\tau_{\mathrm{o}-\mathrm{Ps}}$; (b) $I_{\mathrm{o}-\mathrm{Ps}}$; and (c) variation of the free-volume percentage. The dashed lines are a guide for the eye.

\section{RESULTS}

In Figs. 1, 2(a), and 2(b), the effect of the thickness reduction just after cold rolling on $\tau_{\mathrm{o}-\mathrm{Ps}}$ and $I_{\mathrm{o}-\mathrm{Ps}}$ is plotted, respectively, for PC and PS. The following observations are made by analyzing the figures:

(i) Both $\tau_{\mathrm{o}-\mathrm{Ps}}$ and $I_{\mathrm{o}-\mathrm{Ps}}$ undergo a significant change upon cold rolling in the plastic deformation region. In particular, $\tau_{\mathrm{o}-\mathrm{Ps}}$ increases, whereas a reduction of $I_{\mathrm{o}-\mathrm{Ps}}$ can be observed.

(ii) No significant dependence of the PALS parameters on the thickness reduction appears, provided that the samples are rolled above the yield point as done in this study.

(iii) PC and PS show a similar trend.

Figures 1 and 2(c) show the variation of the free-volume percentage obtained using Eq. (2), with $A=0.00226 \AA^{-3}$ for PC and $A=0.00127 \AA^{-3}$ for PS. These values are derived from the extrapolation of literature data. ${ }^{31}$ As it can be seen $A$ is a material specific parameter, which depends on the chemistry of positronium formation (availability of electrons). The 


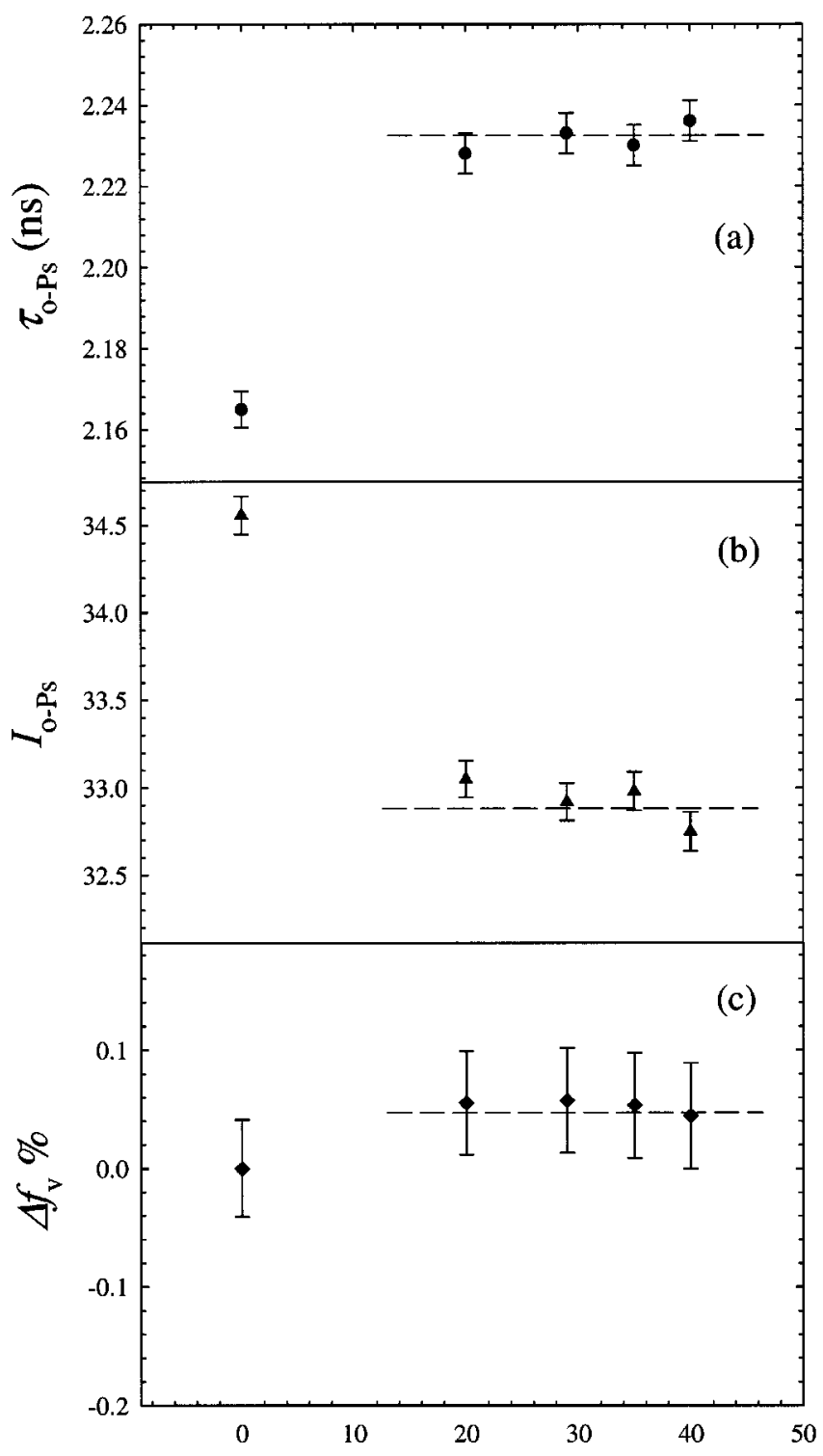

FIG. 2. Effect of the percentage of thickness reduction on the PALS parameters of PS: (a) $\tau_{\mathrm{o}-\mathrm{Ps}}$; (b) $I_{\mathrm{o}-\mathrm{Ps}}$; and (c) variation of the free-volume percentage. The dashed lines are a guide for the eye.

results indicate a free-volume change corresponding to a variation of the specific volume of about $0.2 \%$ for PC. For PS, the free volume seems to increase slightly, although the microstructure must have changed dramatically as shown by the behavior of $\tau_{\mathrm{o}-\mathrm{Ps}}$ and $I_{\mathrm{o}-\mathrm{Ps}}$.

Beside the PALS results, the variation of the tensile modulus before and after cold rolling is shown in Table I. A clear decrease in the tensile modulus of PC and PS for a thickness reduction of, respectively, of $10 \%$ and $30 \%$ can be observed just after cold rolling.

TABLE I. Dynamic tensile moduli of PC and PS before and after cold rolling. Here the thickness reduction is $10 \%$ and $30 \%$ for PC and PS, respectively.

\begin{tabular}{ccc}
\hline \hline Polymer & $E^{\prime}(\mathrm{MPa})$ before rolling & $E^{\prime}(\mathrm{MPa})$ after rolling \\
\hline PC & 2660 & 2540 \\
PS & 3570 & 3475 \\
\hline \hline
\end{tabular}

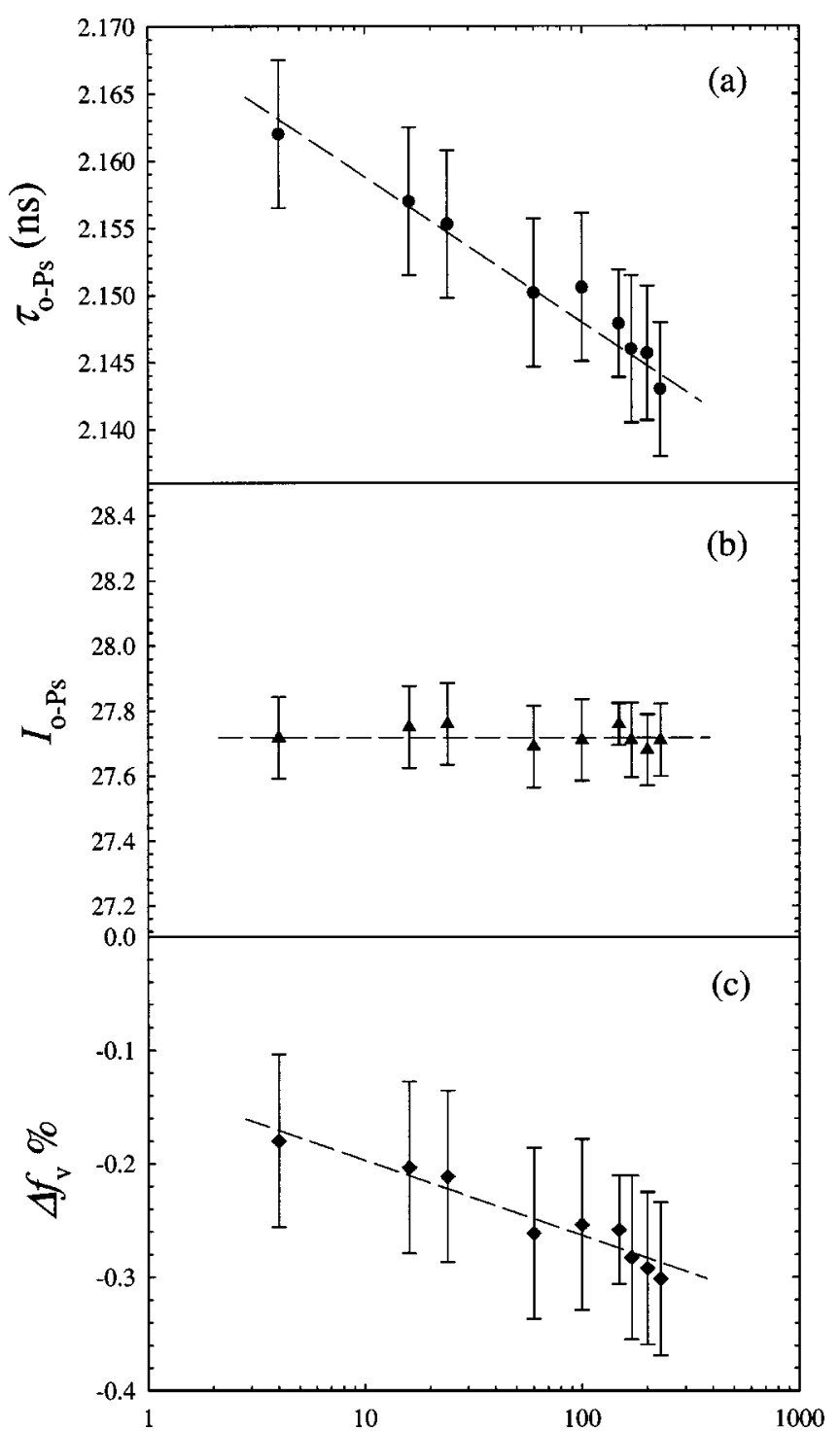

FIG. 3. Time evolution after cold rolling of the PALS parameters of PC: (a) $\tau_{\mathrm{o}-\mathrm{Ps}}$; (b) $I_{\mathrm{o}-\mathrm{Ps}}$; and (c) variation of the free-volume percentage referred to the state before plastic deformation. The dashed lines are a guide for the eye.

The time dependence after cold rolling of both the PALS parameters and tensile modulus was also monitored. Figures $3,4(\mathrm{a})$, and $4(\mathrm{~b})$ show a progressive decrease of $\tau_{\mathrm{o}-\mathrm{Ps}}$, which tends to return to the value before rolling both for PC and PS. On the other hand, the figures show a constant $I_{\mathrm{o}-\mathrm{Ps}}$, within the experimental uncertainty.

From the time evolution of $\tau_{\mathrm{o}-\mathrm{Ps}}$ and $I_{\mathrm{o}-\mathrm{Ps}}$, the variation of the free-volume percentage can be evaluated. As shown in Figs. 3 and 4(c), the free volume evolves towards smaller values than the ones just after rolling, due to the decrease in $\tau_{\text {o-Ps. }}$. In Figs. 3 and 4 we have referred the free-volume variation to the value before rolling. It is worth noting that for PC a densification occurs on systems that have already undergone densification due to the cold rolling. In the case of PS, although cold rolling induces a slight increase in the free volume, the system evolves to a free volume smaller than the one before rolling, which, as pointed out in the Introduction, was not evolving in spite of the larger free volume ("well aged"). This is, at least apparently, in contradiction with 


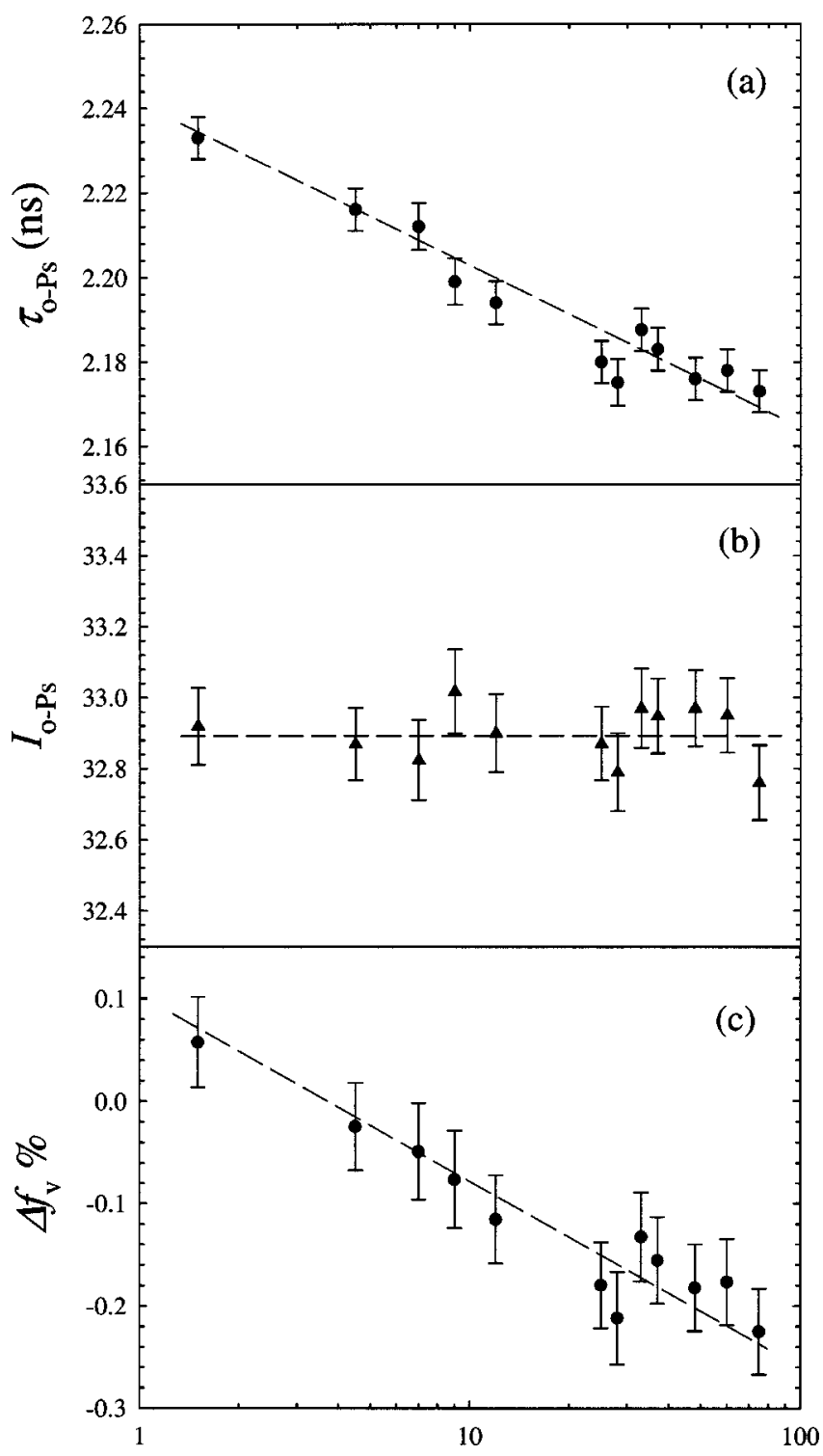

FIG. 4. Time evolution after cold rolling of the PALS parameters of PS: (a) $\tau_{\mathrm{o}-\mathrm{Ps}}$; (b) $I_{\mathrm{o}-\mathrm{Ps}}$; and (c) variation of the free-volume percentage referred to the state before plastic deformation. The dashed lines are a guide for the eye.

simple free-volume concepts, which relate mobility directly to the overall free volume in the system. ${ }^{1,2}$ This point will be discussed in more detail in the following section of this paper.

For the tensile modulus, the same type of time evolution was obtained. Interestingly, the dynamic modulus follows a similar trend to $\tau_{\mathrm{o}-\mathrm{Ps}}$, i.e., it tends to return to its value before rolling in the same time scale as $\tau_{\mathrm{o}-\mathrm{Ps}}$ as shown in Fig. 5. In addition, as pointed out by Van Melick et al., ${ }^{14}$ recovery of the yield stress and strain softening occurs with the same kinetics. In summary, although from the point of view of the mechanical properties, both PC and PS seem to return to their original state, before cold rolling, it is evident from PALS experiments that cold-rolled amorphous glassy polymers have a microstructure that is rather different from the original one.

\section{DISCUSSION}

In the preceding section of the paper, the free volume of PC and PS after plastic deformation was evaluated by means
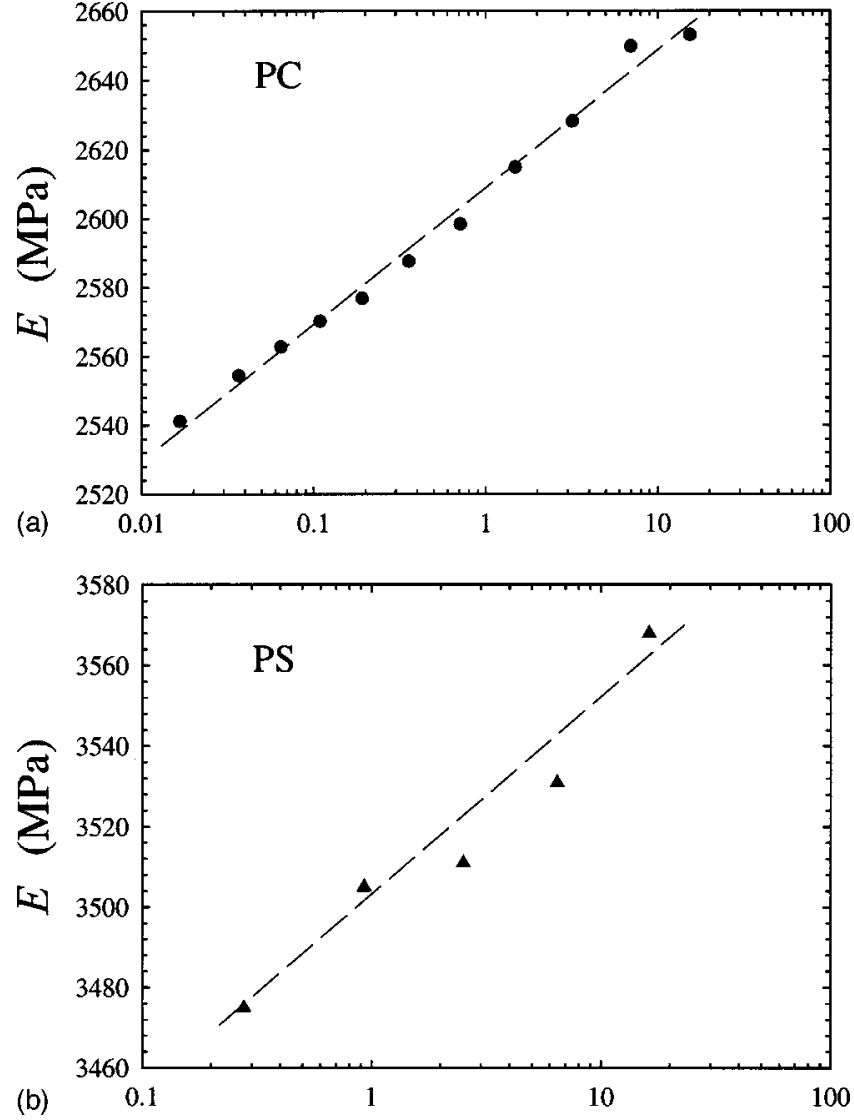

FIG. 5. Time evolution after cold rolling of the dynamic tensile modulus for (a) PC; (b) PS. The dashed lines are a guide for the eye.

of PALS as a combination of size $\left(\tau_{\mathrm{o}-\mathrm{Ps}}\right)$ and concentration of holes $\left(I_{\mathrm{o}-\mathrm{Ps}}\right)$. In this way, we were able to show that plastic deformation of well-aged PC and PS produces an increase in the size of the free-volume holes and a decrease of the concentration of the holes. In previous studies, preformed by some of us on PC, it was shown that physical aging after thermal rejuvenation produces a reduction of the concentration of free volume holes, while the cavity size remains unaltered. $^{26,27}$ This means that a "true" rejuvenation of a well-aged sample should restore the structure to that present before the onset of physical aging, and should hence produce an increase in the concentration of free-volume holes, which means an increasing $I_{\mathrm{o}-\mathrm{Ps}}$ while no changes in $\tau_{\mathrm{o}-\mathrm{Ps}}$ would be expected. It is therefore a straightforward conclusion that, according to our present PALS results, amorphous glassy polymers after plastic deformation have a structure that is qualitatively different from that of a thermally rejuvenated sample. The idea of rejuvenation induced by mechanical deformation as formulated by Struik $^{1,2}$ is clearly not supported by our PALS results. On the other hand, our results do support the hypothesis of an amorphous-amorphous transition, as proposed by McKenna. ${ }^{3}$ We believe that the rejuvenating effect of plastic deformation was misinterpreted due to its ability to reinitiate physical aging similar to thermal rejuvenation. The main feature of the time evolution of the free volume after plastic deformation is the reduction of the cavity size, as testified by the reduction of the $\tau_{\mathrm{o}-\mathrm{Ps}}$ rather than the reduction of the concentration of free-volume holes as is 
the case of physical aging after thermal rejuvenation. ${ }^{26,27}$ In other words, although the macroscopic volume reduction is very similar in both cases, the microscopic mechanism for this free-volume reduction is totally different. In addition, another important observation of the effect produced by plastic deformation is the persistence of the change of the $I_{\mathrm{o}-\mathrm{Ps}}$, which can be considered as a signature of the new amorphous state. From a potential energy landscape point of view, ${ }^{5}$ the new minimum, which the glassy state enters during plastic deformation, corresponds to a situation with a smaller number of free-volume holes.

Our PALS results agree nicely with the change of density reported for PC (Ref. 13) and PS (Ref. 14) after plastic deformation. In particular, Broutman and Patil ${ }^{13}$ found a decrease of the specific volume of $0.25 \%$ for PC and Van Melick et al. ${ }^{14}$ find a very slight decrease of PS specific volume (about $0.03 \%$ ). Comparing these data with our PALS results in Figs. 1 and 2, it looks like our results show a smaller increase in density, since we find a free-volume decrease of $0.2 \%$ for PC and even an increase of $0.05 \%$ for PS.

The change of the dynamic modulus after plastic deformation and its subsequent time evolution is also very interesting, especially when considering the size of the freevolume holes as probed by PALS. Recently, Schmidt et al. ${ }^{32}$ showed that a universal law holds relating the bulk modulus to the volume of the free-volume holes. In particular, the bulk moduli of a large number of polymers were found to decrease linearly with the cavity size. In other words, the macroscopic stiffness of the polymer was found to be related exclusively to the size of the free-volume holes. Although in this work we have employed the dynamic elongation modulus rather than the bulk modulus, a similar correlation was found. In fact, plastic deformation at the same time produces a reduction of the dynamic modulus and an increase of the size of the free-volume holes, i.e., $\tau_{\mathrm{o}-\mathrm{Ps}}$, similar to the findings of Schmidt et al. ${ }^{32}$ This is valid for both PC and PS. During the subsequent time evolution, the modulus increases and the $\tau_{\mathrm{o}-\mathrm{Ps}}$ decreases within the same time scale. From a quantitative point of view, this is shown in Fig. 6 where the volume of the free-volume holes, calculated from $\tau_{\mathrm{o}-\mathrm{Ps}}$ using Eq. (1), is plotted versus the dynamic modulus corresponding to the same time after plastic deformation. In agreement with Schmidt et al $^{32}$ an inverse linear relation is obtained.

It is worth mentioning that in order to get hole volume and dynamic moduli at the same time after plastic deformation, the experimental values of the latter were fitted through an exponential law, which was found to be suitable to describe the time evolution of the dynamic moduli. This allows the dynamic moduli to be calculated at the same time after plastic deformation as in the PALS experiments.

Finally, we would like to comment on the interpretation of our PALS results. First of all it was assumed that Eq. (1), obtained under the assumption of spherical cavities, ${ }^{16}$ is valid even for nonspherical ones, which could in principle be present in samples subjected to plastic deformation via cold rolling. This procedure can induce orientation in the polymer sample, and could also give rise to the formation of nonspherical cavities. However, experimentally we have found that injection moulded amorphous polymer samples, which were clearly oriented from the observed birefringence, did not show significant differences in the $\tau_{\mathrm{o}-\mathrm{Ps}}$ compared to similar samples free of orientation. This means that Eq. (1) probably is a good approximation even for nonspherical cavities, at least for the strains applied in the present situation.

Another point for discussion is the structural significance of $I_{\mathrm{o}-\mathrm{Ps}}$. Recently, it was observed that application of large stresses to amorphous glassy polymers can lead to the formation of free radicals, which would inhibit positronium formation and, therefore, lead to a reduction in the $I_{\mathrm{o}-\mathrm{Ps}}{ }^{33} \mathrm{How}-$ ever, our experimental conditions are much less drastic than those as reported by Günther-Schade $e t$ al. ${ }^{33}$ However, even if a certain amount of radicals are present, we believe that they do not affect $I_{\mathrm{o}-\mathrm{Ps}}$ for three reasons:

(i) Though we do not have any direct experimental evidence, we can postulate that the concentration of radicals should increase with the amount of deformation applied to the samples upon cold rolling and this would lead to a reduction of $I_{\mathrm{o}-\mathrm{Ps}}$ dependent on the plastic deformation. In our case, $I_{\mathrm{o}-\mathrm{Ps}}$ is independent of the plastic deformation, as shown in the Results section;

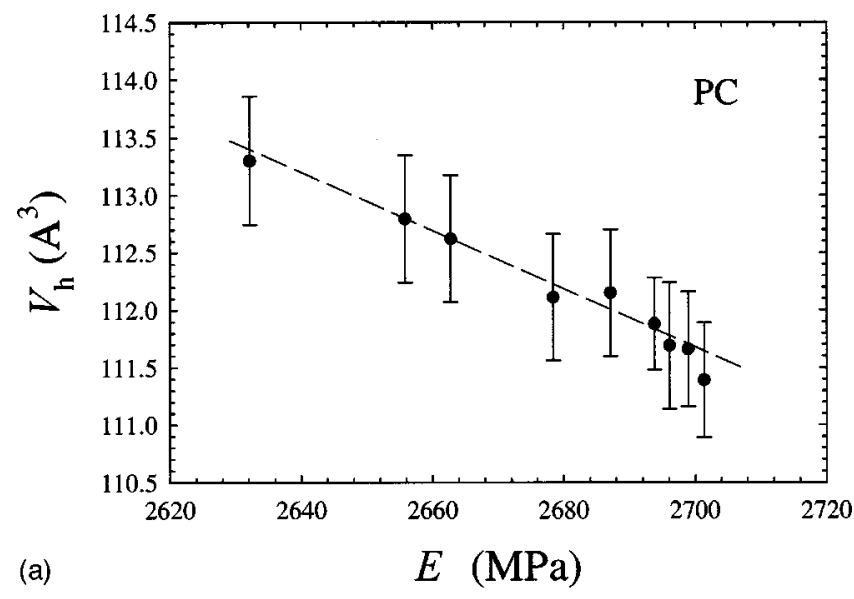

(a)

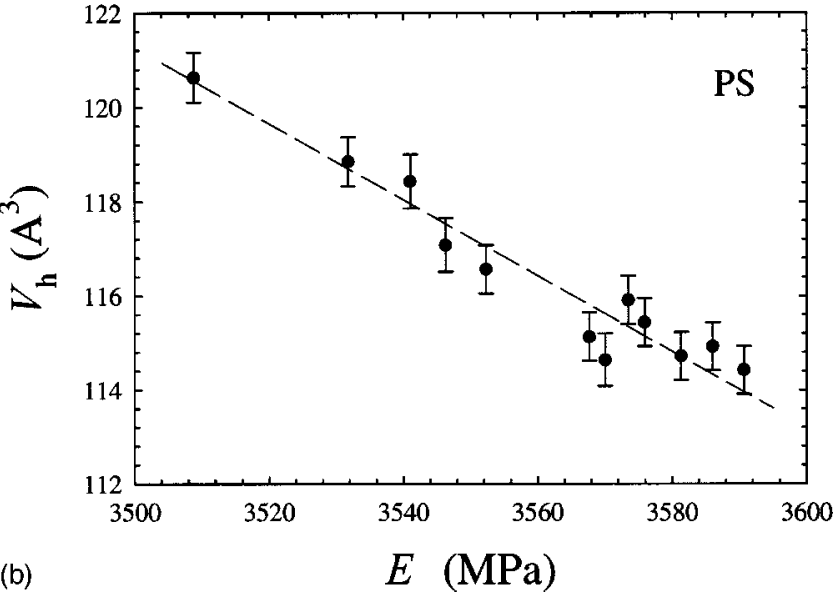

FIG. 6. Relation between the hole volume and the dynamic tensile modulus during the time evolution after cold rolling. The values of the dynamic tensile modulus are calculated from the exponential fitting of the experimental values of the dynamic modulus with time. The dashed lines are a guide for the eye. 
(ii) Though in the glassy state, radicals would slowly disappear with time and $I_{\mathrm{o}-\mathrm{Ps}}$ would go up. That does not occur and, instead, $I_{\mathrm{o}-\mathrm{Ps}}$ stays at the new value after cold rolling indefinitely;

(iii) As already discussed, the variation of the free volume evaluated from both $I_{\mathrm{o}-\mathrm{Ps}}$ and $\tau_{\mathrm{o}-\mathrm{Ps}}$ resembles the variation of the specific volume evaluated using macroscopic techniques. ${ }^{13,14}$

These observations support our interpretation of $I_{\mathrm{o}-\mathrm{Ps}}$ as a factor that provides significant information on the polymer structure, at least if the experimental procedures are carefully executed to avoid various possible artifacts.

\section{CONCLUSIONS}

The effect of plastic deformation induced by cold rolling of glassy PC and PS has been investigated by means of PALS and dynamic-mechanical spectroscopy, which yields information on the time dependent evolution of the freevolume microstructure and the dynamic moduli. An increase of the size of the free-volume holes and a decrease of their concentration is observed as a consequence of plastic deformation. The subsequent time evolution of the free-volume proceeds via a reduction of the size of the free-volume holes towards the value that occurred before plastic deformation, while the concentration of free-volume holes remained constant. These results indicate that plastic deformation provokes an amorphous-amorphous transition in amorphous glassy polymers similar to the ones occurring in other glassy systems like silica. On the other hand, the idea of a mechanical rejuvenation, which arose from the experimental observation of reinitiated physical aging, is not supported by our PALS results. In fact, the time evolution of the free volume after plastic deformation suggests a totally different microstructure of the free volume compared to the structure after thermal rejuvenation, which consists of the reduction of the concentration of free-volume holes. Finally, the dynamic modulus after plastic deformation and its subsequent time evolution shows a clear relation to the change in volume of the free-volume holes, indicating that the stiffness of amorphous glassy polymers can be fully attributed to the freevolume structure as probed by PALS.

\section{ACKNOWLEDGMENTS}

The work of D.C. and S.J.P. forms part of the research program of the Dutch Polymer Institute (DPI). This work is carried out under DPI Project No. 285: "Influence of Applied Stress and Physical Ageing on Diffusion and Solubility of Oxygen and Anti-Oxidants." The authors would like to thank
C. J. M. Meesters and L. E. Govaert of the department of Mechanical Engineering at Eindhoven University of Technology for their assistance in the mechanical characterization of the rejuvenated samples.

${ }^{1}$ L. C. E. Struik, Physical Aging in Amorphous Glassy Polymers and Other Materials (Elsevier, Amsterdam, 1978).

${ }^{2}$ L. C. E. Struik, Polymer 38, 4053 (1997).

${ }^{3}$ G. B. McKenna, J. Phys.: Condens. Matter 15, 737 (2003).

${ }^{4}$ M. Aboulfaraj, C. Gsell, D. Mangelink, and G. B. McKenna, J. Non-Cryst. Solids 172-174, 615 (1994).

${ }^{5}$ F. H. Stillinger, Science 267, 1935 (1995).

${ }^{6}$ D. L. Malandro and D. J. Lacks, J. Chem. Phys. 110, 4593 (1999).

${ }^{7}$ G. Gagnon, J. Patton, and D. J. Lacks, Phys. Rev. E 64, 051508 (2001).

${ }^{8}$ T. A. Weber and F. H. Stillinger, J. Chem. Phys. 80, 2742 (1984).

${ }^{9}$ D. J. Lacks, Phys. Rev. Lett. 80, 5385 (1998).

${ }^{10}$ D. J. Lacks, Phys. Rev. Lett. 84, 4629 (2000).

${ }^{11}$ V. V. Brazhkin and A. G. Lyapin, J. Phys.: Condens. Matter 15, 6059 (2003), and references therein.

${ }^{12}$ S. S. Jang and W. H. Jo, J. Chem. Phys. 110, 7524 (1999).

${ }^{13}$ J. L. Broutman and R. S. Patil, Polym. Eng. Sci. 11, 165 (1971).

${ }^{14}$ H. G. H. Van Melick, L. E. Govaert, B. Raas, W. J. Nauta, and H. E. H. Meijer, Polymer 44, 1171 (2003).

${ }^{15}$ O. E. Mogensen, Positron Annihilation in Chemistry (Springer, Berlin, 1995).

${ }^{16}$ M. Eldrup, D. Lightbody, and J. N. Sherwood, Chem. Phys. 63, 51 (1981).

${ }^{17}$ V. P. Shantarovich and J. Radioanal, J. Radioanal. Nucl. Chem. 210, 357 (1996).

${ }^{18}$ C. Wastlund and F. H. J. Maurer, Polymer 39, 2897 (1998).

${ }^{19}$ M. Welander and F. H. J. Maurer, Mater. Sci. Forum 105-110, 1811 (1992).

${ }^{20}$ X. S. Li and M. C. Boyce, J. Polym. Sci., Part B: Polym. Phys. 31, 869 (1993).

${ }^{21}$ D. Cangialosi, H. Schut, M. Wübbenhorst, J. van Turnhout, and A. van Veen, Radiat. Phys. Chem. 68, 507 (2003).

${ }^{22}$ C. L. Wang, K. Hirata, J. Kawahara, and Y. Kobayashi, Phys. Rev. B 58, 14864 (1998).

${ }^{23}$ A. Alba García, L. D. A. Siebbeles, H. Schut, and A. van Veen, Radiat. Phys. Chem. 68, 515 (2003).

${ }^{24}$ J. Zrubcova, J. Kristiak, W. B. Pedersen, N. J. Pedersen, and M. Eldrup, Mater. Sci. Forum 363, 359 (2001).

${ }^{25}$ F. H. J. Maurer and M. Schmidt, Radiat. Phys. Chem. 58, 509 (2000).

${ }^{26}$ D. Cangialosi, H. Schut, A. van Veen, and S. J. Picken, Macromolecules 36, 142 (2003).

${ }^{27}$ D. Cangialosi, M. Wübbenhorst, H. Schut, A. van Veen, and S. J. Picken, Phys. Rev. B 69, 134206 (2004).

${ }^{28}$ O. A. Hasan, M. C. Boyce, X. S. Li, and S. Berko, J. Polym. Sci., Part B: Polym. Phys. 31, 185 (1993).

${ }^{29}$ P. Kirkegaard, M. Eldrup, O. E. Mogensen, and N. J. Pedersen, Comput. Phys. Commun. 23, 307 (1981).

${ }^{30}$ C. Dauwe, B. van Waeyenberge, and N. Balcaen, Phys. Rev. B 68, 132202 (2003); 445-446, 229 (2004).

${ }^{31}$ K. Hagiwara, T. Ougizawa, T. Inoue, K. Hirata, and Y. Kobayashi, Radiat. Phys. Chem. 58, 525 (2000).

${ }^{32}$ M. Schmidt, M. Olsson, and F. H. J. Maurer, J. Chem. Phys. 112, 11095 (2000).

${ }^{33}$ K. Günther-Schade, H. L. Castricum, H. J. Ziegler, H. Bakker, and F. Faupel, Polym. Eng. Sci. 44, 1351 (2004). 